\title{
Quantum Hall-MHD equations for a non-uniform dense magnetoplasma with electron temperature anisotropy
}

\author{
P. K. SHUKLA $A^{1,2} \dagger$ and L. STENFL $\mathrm{O}^{1,2} \ddagger$ \\ ${ }^{1}$ Institut für Theoretische Physik IV, Fakultät für Physik und Astronomie, \\ Ruhr-Universität Bochum, D-44780 Bochum, Germany \\ (ps@tp4.rub.de) \\ ${ }^{2}$ Department of Physics, Umeå University, SE-90187 Umeå, Sweden \\ (lennart.stenflo@physics.umu.se)
}

(Received 14 March 2008 and in revised form 2 April 2008, first published online 23 May 2008)

\begin{abstract}
Nonlinear quantum Hall-MHD equations for a warm dense magnetoplasma with an anisotropic electron pressure are derived. The nonlinear equations include the quantum force associated with electron tunneling effects. The newly found equations can be used to investigate the dense plasma stability, as well as different types of waves, instabilities, and nonlinear structures in a warm dense magnetoplasma.
\end{abstract}

In a dense quantum Fermi plasma, the electrons are degenerate and the corresponding electron distribution obeys Fermi-Dirac statistics. In such a dense plasma, the governing quantum kinetic equation $[1,2]$, deduced from the Wigner equation [3], accounts for the electron degeneracy. In the past, several authors have studied the linear properties of electrostatic waves in unmagnetized $[4,5]$ and magnetized [6-8] plasmas containing degenerate electrons.

Recently, there has been a renewed interest in investigating waves [9-14], instabilities $[15,16]$ and turbulence [17], as well as coherent nonlinear structures [18-20] at nanoscales in dense quantum plasmas. The latter are ubiquitous in astrophysical bodies [21-23] (e.g. the interior of white dwarfs, magnetars, supernovae, etc.) and in intense laser-solid density plasma interaction experiments [24-26].

In this letter, we present nonlinear quantum Hall-MHD equations for a warm dense magnetoplasma in which the degenerate electrons have a pressure anisotropy. This may arise when the electrons are heated by intense electromagnetic and neutron beams in a dense magnetoplasma.

$\dagger$ Also at School of Physics, University of KwaZulu-Natal, 4000 Durban, South Africa; Max-Planck-Institut für extraterrestrische Physik, D-85741 Garching, Germany; Grupo de Lasers e Plasmas/Instituto de Plasmas e Fusao Nuclear, Instituto Superior Técnico, Universidade Técnica de Lisboa, 1049-001 Lisboa, Portugal; CCLRC Centre for Fundamental Physics, Rutherford Appleton Laboratory, Chilton, Didcot, Oxon 0X11 0QX, UK; SUPA, Department of Physics, University of Strathclyde, Glasgow G4 0NG, UK; Department of Physics, CIIT, Islamabad, Pakistan.

f Also at Department of Physics, Linköping University, SE-58183 Linköping, Sweden. 
Let us suppose that our dense Fermionic plasma is embedded in an external magnetic field $\hat{\mathbf{z}} B_{0}$, where $\hat{\mathbf{z}}$ is a unit vector along the $z$-axis, and $B_{0}$ is the strength of the magnetic field. In the presence of electromagnetic fields, the dynamics of the inertialess hot electrons is governed by

$$
0=-n_{\mathrm{e}} e\left(\mathbf{E}+\frac{1}{c} \mathbf{u}_{\mathrm{e}} \times \mathbf{B}\right)-\nabla \cdot \mathscr{P}+n_{\mathrm{e}} \mathbf{F}_{\mathrm{Q}}
$$

where $n_{\mathrm{e}}$ is the electron number density, $\mathbf{u}_{\mathrm{e}}$ is the electron fluid velocity, $\mathbf{E}$ and $\mathbf{B}$ are the electric and magnetic fields, respectively, $e$ is the magnitude of the electron charge, $c$ is the speed of light in vacuum, $\mathscr{P}=P_{\perp} \mathbf{I}+\left(P_{\|}-P_{\perp}\right) \hat{\mathbf{b}} \hat{\mathbf{b}}$ is the electron pressure, $\mathbf{I}$ is the unit dyad, and $\hat{\mathbf{b}} \hat{\mathbf{b}}=\mathbf{B B} / B^{2}$ is the dyad formed from the unit vector $\hat{\mathbf{b}}$. Here $P_{\|}$and $P_{\perp}$ are the components of the electron pressure parallel and perpendicular to $\hat{\mathbf{b}}$, respectively. Furthermore, the quantum force associated with the quantum Bohm potential is [27]

$$
\mathbf{F}_{\mathrm{Q}}=\frac{\hbar^{2}}{2 m_{\mathrm{e}}} \nabla\left(\frac{\nabla^{2} \sqrt{n_{\mathrm{e}}}}{\sqrt{n_{\mathrm{e}}}}\right),
$$

where $\hbar$ is the Planck constant divided by $2 \pi$, and $m_{\mathrm{e}}$ is the electron mass. For low-phase velocity (in comparison with $c$ ) disturbances, the electromagnetic fields are determined from the Faraday law

$$
\frac{\partial \mathbf{B}}{\partial t}=-c \nabla \times \mathbf{E}
$$

and Ampère's law

$$
\nabla \times \mathbf{B}=\frac{4 \pi e}{c}\left(Z_{\mathrm{i}} n_{\mathrm{i}} \mathbf{u}_{\mathrm{i}}-n_{\mathrm{e}} \mathbf{u}_{\mathrm{e}}\right),
$$

where $Z_{\mathrm{i}}$ is the ion charge state, and $n_{\mathrm{i}}$ and $\mathbf{u}_{\mathrm{i}}$ are the ion number density and the ion fluid velocity, respectively. The latter are determined from the ion continuity and momentum equations

and

$$
\frac{d n_{\mathrm{i}}}{d t}+n_{\mathrm{i}} \nabla \cdot \mathbf{u}_{\mathrm{i}}=0
$$

$$
\rho_{\mathrm{i}} \frac{d \mathbf{u}_{\mathrm{i}}}{d t}=Z_{\mathrm{i}} n_{\mathrm{i}} e\left(\mathbf{E}+\frac{1}{c} \mathbf{u}_{\mathrm{i}} \times \mathbf{B}\right)+\rho_{\mathrm{i}} \mathbf{g}+2 \rho_{\mathrm{i}} \mathbf{u}_{\mathrm{i}} \times \mathbf{\Omega},
$$

where $d / d t=(\partial / \partial t)+\mathbf{u}_{\mathrm{i}} \cdot \nabla, \rho_{\mathrm{i}}=n_{\mathrm{i}} m_{\mathrm{i}}$ is the ion mass density, $m_{\mathrm{i}}$ is the ion mass, $\rho_{\mathrm{i}} \mathbf{g}$ is the gravity force, and $|\boldsymbol{\Omega}|$ is the angular rotation frequency of the ions. The quantum force acting on the ions is much smaller than that on the electrons, and therefore it is not included in (6). Since in dense quantum plasmas, the ions are much colder than the electrons, we have also neglected the anisotropic ion pressure.

We now eliminate $\mathbf{E}$ from (6) by using (1), obtaining

$$
\begin{aligned}
\rho_{\mathrm{i}} \frac{d \mathbf{u}_{\mathrm{i}}}{d t}= & \frac{e Z_{\mathrm{i}} n_{\mathrm{i}}}{c}\left(1-\frac{Z_{\mathrm{i}} n_{\mathrm{i}}}{n_{\mathrm{e}}}\right) \mathbf{u}_{\mathrm{i}} \times \mathbf{B} \\
& +\frac{Z_{\mathrm{i}} n_{\mathrm{i}}}{n_{\mathrm{e}}}\left[\frac{(\nabla \times \mathbf{B}) \times \mathbf{B}}{4 \pi}-\nabla \cdot \mathscr{P}\right]+\rho_{\mathrm{i}} \mathbf{g}+2 \rho_{\mathrm{i}} \mathbf{u}_{\mathrm{i}} \times \mathbf{\Omega}+Z_{\mathrm{i}} n_{\mathrm{i}} \mathbf{F}_{\mathrm{Q}},
\end{aligned}
$$

where from (4) we used

$$
\mathbf{u}_{\mathrm{e}}=\frac{Z_{\mathrm{i}} n_{\mathrm{i}}}{n_{\mathrm{e}}} \mathbf{u}_{\mathrm{i}}-\frac{c \nabla \times \mathbf{B}}{4 \pi e n_{\mathrm{e}}}
$$


By using the identity

$$
(\nabla \times \mathbf{B}) \times \mathbf{B}=\left[(\mathbf{B} \cdot \nabla) \mathbf{B}-\frac{1}{2} \nabla B^{2}\right]
$$

we can express (7) as

$$
\begin{aligned}
\rho_{\mathrm{i}} \frac{d \mathbf{u}_{\mathrm{i}}}{d t}= & \frac{e Z_{\mathrm{i}} n_{\mathrm{i}}}{c}\left(1-\frac{Z_{\mathrm{i}} n_{\mathrm{i}}}{n_{\mathrm{e}}}\right) \mathbf{u}_{\mathrm{i}} \times \mathbf{B}-\frac{Z_{\mathrm{i}} n_{\mathrm{i}}}{n_{\mathrm{e}}} \nabla\left(P_{\perp}+\frac{B^{2}}{8 \pi}\right) \\
& +\frac{Z_{\mathrm{i}} n_{\mathrm{i}}}{n_{\mathrm{e}}} \frac{(\mathbf{B} \cdot \nabla)}{4 \pi}\left[\mathbf{B}-\frac{4 \pi\left(P_{\|}-P_{\perp}\right) \mathbf{B}}{B^{2}}\right]+\rho_{\mathrm{i}} \mathbf{g}+2 \rho_{\mathrm{i}} \mathbf{u}_{\mathrm{i}} \times \mathbf{\Omega}+Z_{\mathrm{i}} n_{\mathrm{i}} \mathbf{F}_{\mathrm{Q}},
\end{aligned}
$$

where we have introduced $\nabla \cdot \mathscr{P}=\nabla P_{\perp}+(\mathbf{B} \cdot \nabla)\left[\left(P_{\|}-P_{\perp}\right) \mathbf{B} / B^{2}\right]$.

Furthermore, by using (1) and (4) we can write (3) as

$$
\frac{\partial \mathbf{B}}{\partial t}=\nabla \times\left[\left(\frac{Z_{\mathrm{i}} n_{\mathrm{i}}}{n_{\mathrm{e}}} \mathbf{u}_{\mathrm{i}}-\frac{c \nabla \times \mathbf{B}}{4 \pi e n_{\mathrm{e}}}\right) \times \mathbf{B}\right]+\frac{c}{e} \nabla \times\left(\frac{\nabla \cdot \mathscr{P}}{n_{\mathrm{e}}}\right) .
$$

Let us invoke the following Chew, Goldberger and Low equations [28] for the parallel and perpendicular components of the electron pressure

$$
\left(\frac{\partial}{\partial t}+\mathbf{u}_{\mathrm{e}} \cdot \nabla\right) P_{\|}+P_{\|} \nabla \cdot \mathbf{u}_{\mathrm{e}}+2 P_{\|}(\hat{\mathbf{b}} \hat{\mathbf{b}} \cdot \nabla) \cdot \mathbf{u}_{\mathrm{e}}=0,
$$

and

$$
\left(\frac{\partial}{\partial t}+\mathbf{u}_{\mathrm{e}} \cdot \nabla\right) P_{\perp}+2 P_{\perp} \nabla \cdot \mathbf{u}_{\mathrm{e}}-P_{\perp}(\hat{\mathbf{b}} \hat{\mathbf{b}} \cdot \nabla) \cdot \mathbf{u}_{\mathrm{e}}=0
$$

where $\mathbf{u}_{\mathrm{e}}$ is given by $(8)$.

Equations (5) and (9)-(13), which form a closed system under the quasi-neutrality condition [29] $e\left(Z_{\mathrm{i}} n_{\mathrm{i}}-n_{\mathrm{e}}\right)=-q_{\mathrm{d}} n_{\mathrm{d}}$, are the desired quantum Hall-MHD equations. Here $q_{\mathrm{d}} n_{\mathrm{d}}$ is the charge density of dust grains (if present in a non-uniform dense magnetoplasma). In the absence of charged dust grains the condition $Z_{\mathrm{i}} n_{\mathrm{i}}=n_{\mathrm{e}} \equiv n$ would suffice.

In a quasi-neutral unmagnetized isotropic plasma without dust grains, we have from $(10)$

$$
\frac{d \mathbf{u}_{\mathrm{i}}}{d t}=-\frac{\nabla P}{\rho}+\mathbf{g}+2 \mathbf{u}_{\mathrm{i}} \times \mathbf{\Omega}+\frac{Z_{\mathrm{i}} \hbar^{2}}{2 m_{\mathrm{e}} m_{\mathrm{i}}} \nabla\left(\frac{\nabla^{2} \sqrt{n}}{\sqrt{n}}\right),
$$

where $P$ is the isotropic electron pressure and $\rho=n m_{\mathrm{i}}$. In an isotropic dense plasma, we also have the adiabatic equation of state

$$
\frac{d}{d t}\left(\frac{P}{\rho^{\gamma}}\right)=0
$$

where $\gamma$ is the adiabatic index.

Equations (14) and (15) are similar in form to the equations describing solitary atmospheric waves [30]. Thus, linearizing (14) and (15) around the equilibrium values (namely $\rho_{0}$ and $P_{0}$ ), we obtain the dispersion relation for a non-uniform plasma

$$
\omega^{2}=\omega_{\mathrm{g}}^{2} \frac{k_{\perp}^{2}}{k^{2}}+\frac{4}{k^{2}}(\mathbf{k} \cdot \mathbf{\Omega})^{2}
$$

where $\omega$ is the frequency, $\omega_{\mathrm{g}}=\left[\left(d_{z} \rho_{0} / \rho_{0}-d_{z} P_{0} / \gamma P_{0}\right) d_{z} P_{0} / \rho_{0}\right]^{1 / 2}$, and $k_{\perp}^{2}=$ $k^{2}-k_{z}^{2}$. Here $k_{\perp}$ and $k_{z}$ are the wave numbers across and along the density and pressure inhomogeneities, namely $d_{z} \rho_{0}$ and $d_{z} P_{0}$. The equilibrium, without ion flow, 
is determined from

$$
g=-\frac{d_{z} P_{0}}{\rho_{0}}+\frac{Z_{\mathrm{i}} \hbar^{2}}{2 m_{\mathrm{e}} m_{\mathrm{i}}} \frac{d}{d z}\left(\frac{d_{z}^{2} \sqrt{\rho_{0}}}{\sqrt{\rho_{0}}}\right),
$$

where $\mathbf{g}=-g \hat{\mathbf{z}}$ and $\hat{\mathbf{z}}$ is the unit vector along the $z$-axis in a Cartesian coordinate system.

Following Stenflo [31], the dynamical equations for nonlinearly interacting modes in our inhomogeneous dense unmagnetized plasma are

$$
\frac{\partial \nabla^{2} u_{z}}{\partial t}-\hat{\mathbf{z}} \cdot\left(\nabla \times \nabla \times \mathbf{u}_{\mathrm{i}} \cdot \nabla \mathbf{u}_{\mathrm{i}}\right)+\nabla_{\perp}^{2} \chi+R=0,
$$

and

$$
\frac{d \chi}{d t}-\omega_{\mathrm{g}}^{2} u_{z}=0
$$

where $u_{z}=\hat{\mathbf{z}} \cdot \mathbf{u}_{\mathrm{i}}, \chi=(1 / \rho)-\left(1 / \rho_{0}\right)$, and $R=2 \hat{\mathbf{z}} \cdot\left(\nabla \times \nabla \times\left(\mathbf{u}_{\mathrm{i}} \times \mathbf{\Omega}\right)\right)$. Here $\chi$ has been normalized by the factor $d_{z} P_{0}$. In two-space dimensions, the coupled equations (18) and (19) with $R=0$ (namely no ion rotation) admit a stationary dipolar vortex [31,32] (a modon) whose profile is presented in [31].

In summary, we have presented the nonlinear quantum Hall-MHD equations in a non-uniform dense magnetoplasma with degenerate electrons having an anisotropic pressure. These equations can be used to investigate different kinds of waves, anisotropic electron pressure driven instabilities, as well as multiscale turbulence and structures in non-uniform dense magnetoplasmas. As an illustration, we have presented an example of linear and nonlinear electrostatic waves in non-uniform unmagnetized plasmas containing electron density and electron pressure gradients. It turns out that the quantum effects appear through the equilibrium state in the analyses of linear and nonlinear electrostatic modes in a non-uniform dense Fermi plasma.

\section{References}

[1] Klimontovich, Yu. L. and Silin, V. P. 1952 ZhETF 23, 151.

[2] Kon'kov, V. L., Gorshenko, V. N. and Bogdanov, E. P. 1967 Izvestiya VUZ Fizika 10, 71.

[3] Wigner, E. 1932 Phys. Rev. 40, 749.

[4] Bohm, D. and Pines, D. 1953 Phys. Rev. 92, 609.

[5] Pines, D. 1961 Plasma Phys. 2, 2.

[6] Kelley, D. C. 1964 Phys. Rev. 134, A641.

[7] Oberman, C. and Ron, A. 1963 Phys. Rev. 130, 1291.

[8] Kuzelev, M. V. and Rukhadze, A. A. 1999 Phys. Usp. 42, 603.

[9] Maafa, N. 1993 Phys. Scr. 48, 351.

[10] Melrose, D. B. and Weise, J. I. 2002 Phys. Plasmas 9, 4473.

[11] Shukla, P. K. 2007 Phys. Lett. A 369, 312.

[12] Ren, H., Wu, Z. and Chu, P. K. 2007 Phys. Plasmas 14, 062102.

[13] Marklund, M. and Brodin, G. 2007 Phys. Rev. Lett. 98, 025001.

[14] Brodin, G. and Marklund, M. 2007 Phys. Rev. E 76, 055403.

[15] Manfredi, G. 2005 Fields Inst. Comm. 46, 263.

[16] Shukla, P. K. and Stenflo, L. 2006 Phys. Plasmas 13, 044505.

[17] Shaikh, D. and Shukla, P. K. 2007 Phys. Rev. Lett. 99, 125002.

[18] Shukla, P. K. and Eliasson, B. 2006 Phys. Rev. Lett. 96, 245001. 
[19] Shukla, P. K. and Eliasson, B. 2007 Phys. Rev. Lett. 99, 096401.

[20] Brodin, G. and Marklund, M. 2007 Phys. Plasmas 14, 112107.

[21] Chabrier, G., Douchin, F. and Potekhin, A. Y. 2002 J. Phys.: Condens. Matter 14, 9133.

[22] Chabrier, G., Saumon, D. and Potekhin, A. Y. 2006 J. Phys. A: Math. Gen. 39, 4411.

[23] Harding, A. K. and Lai, D. 2006 Rep. Prog. Phys. 69, 2631.

[24] Marklund, M. and Shukla, P. K. 2006 Rev. Mod. Phys. 78, 591.

[25] Salamin, Y. I., Hu, S. X., Hatsagortsyan, K. Z. and Keitel, C. H. 2006 Phys. Rep. 427, 41.

[26] Glenzer, S. H. et al. 2007 Phys. Rev. Lett. 98, 065002.

[27] Gardner, C. L. and Ringhofer, C. 1996 Phys. Rev. E 53, 157.

[28] Bittencourt, J. A. 1986 Fundamentals of Plasma Physics. New York: Pergamon.

[29] Bharuthram, R., Saleem, H. and Shukla, P. K. 1992 Phys. Scr. 45, 512.

Shukla, P. K. and Rao, N. N. 1996 Phys. Plasmas 3, 1770.

Birk, G. T., Kopp, A. and Shukla, P. K. 1996 Phys. Plasmas 3, 3564.

Mamun, A. A. and Shukla, P. K. 2001 Phys. Lett. A 290, 173.

Shukla, P. K. and Mamun, A. A. 2003 New J. Phys. 5, 17.

[30] Stenflo, L. 1991 Phys. Scr. 43, 599.

[31] Stenflo, L. 1987 Phys. Fluids 30, 3297.

[32] Shukla, P. K., Birk, G. T. and Bingham, R. 1995 Geophys. Res. Lett. 22, 671. 